\title{
Optical image encryption using gamma distribution phase masks in the gyrator domain
}

\author{
Wenqing Sun ${ }^{*} \mathbb{D}$, Lei Wang, Jun Wang, Hua Li and Quanying Wu
}

\begin{abstract}
Background: Optical information encryption technology has received extensive attention from researchers in recent years because of its advantages of parallel and high-speed processing capability, as well as the controllability of phase components.
\end{abstract}

Methods: An encryption method for grayscale images with a pair of random phase masks based on gamma distribution in the gyrator domain is proposed. In this scheme, two random distribution phase-images using random parameters according to the definition of the gamma probability distribution function are generated. They are loaded onto spatial light modulators as random phase masks used in the encryption process. The input grayscale image transmitted through the first random phase mask. And then they are encoded by the first gyrator transform. The resulting information is again encrypted by a second gamma distribution random phase mask at the gyrator frequency plane. The final results are encoded by the second gyrator transform.

Results: Numerical simulations are presented to confirm the security, validity, and flexibility of the proposed idea. The gyrator transform rotation angle sensitivity test is also simulated. The occlusion and noise attacks analysis establish the scheme's robustness.

Conclusions: In gyrator transform-based optical image encryption cipher system, encrypting the input image with different parameters of gamma distribution RPMs will significantly change the statistical distribution of phase in the ciphertext. It means that the phase distribution in the ciphertext will not obey the law of random scattering medium. Therefore, it has potential to resist the attack based on the phase retrieval algorithm. Therefore, the security and flexibility of encryption can be improved by using gamma distribution RPMs.

Keywords: Optical encryption, Gyrator transform, Random phase mask, Gamma distribution

\section{Introduction}

Optical information encryption technology has received extensive attention from researchers in recent years because of its advantages of parallel and high-speed processing capability, as well as the controllability of phase components. One of the earliest and most widely studied optical encryption method for image encryption was the double random phase encoding (DRPE) system which was proposed by Refregier and Javidi [1-5]. This

\footnotetext{
* Correspondence: sunwenqing@mail.usts.edu.cn

Jiangsu Key Laboratory of Micro and Nano Heat Fluid Flow Technology and Energy Application, School of Mathematics and Physics, Suzhou University of Science and Technology, Suzhou, Jiangsu 215009, People's Republic of China
}

architecture is based on a 4-f imaging system and together with a pair of independent random phase keys (PRKs), which one in the input plane and another in the Fourier plane for encryption amplitude image. After more than 20 years of development, the family of optical information encryption systems has been greatly expanded. They provide many degrees of freedom with which optical beam may be encoded, including amplitude, phase, wavelength, and polarization. Now, there are still many examples showing that optical security and encoding techniques have continued to attract the attention of researchers [6-8]. 
A number of optical encryption methods have been proposed, based on the conventional DRPE configuration, to further enlarge the key space of the encryption system by extending it to the fractional Fourier transform (FrFT) domain [9-11], Hartley transform domain [12, 13], Fresnel domain [14-16] and wavelet domain $[17,18]$ et al. The gyrator transform (GT) belongs to the linear canonical integral transform and corresponds to the rotations in the twisted position-spatial frequency planes of phase space $[19,20]$. This scheme is flexible and easy to implement through an optoelectronic system. Therefore, many improved solutions have been proposed for color image [2125], multiple images [26-30], and watermarking [31], etc.

For security enhancement, another alternative is to use structured phase key (SPK) in the encryption-decryption process instead of white-noised RPK. Several kinds of SPK have been constructed, such as fractal zone mask [32], linear phase mask [33], toroidal zone mask [3436], spiral phase mask [37-44], etc. All these SPKs have shown significant simplicity and robustness to meet the requirements of high flexibility and safety. They have been applied to different domains mentioned above.

In this paper, an image encryption and decryption based on DRPE and gamma distribution phase masks in GT domain is introduced. In this scheme, two random distribution phase-images are generated using random parameters according to the definition of the gamma probability distribution function. They are loaded onto spatial light modulators (SLMs) as RPKs used in encryption process. A grayscale image is encrypted with a first RPK placed at the input plane and then performed the first GT. The resulting spectrum is again encrypted into second RPK placed at the GT spectrum plane and then executed the second GT. The construction parameters of RPKs and rotation angles of GT are introduced for encryption. Therefore, the security and flexibility of the DRPE system are enhanced.

The paper is organized as follows: In Section 2, we present a brief mathematical description of GT, gamma distribution, and the encryption scheme. In Section 3, we present the results based on computer simulations for validation and evaluation of the scheme's performance. Finally, the conclusions of the study are summarized in Section 4.

\section{Methods}

\section{Gyrator transform}

The GT is a linear canonical integral transform and similar to the FrFT. It produces rotation in the twisted position spatial frequency planes. For a two-dimensional function $f(x, y)$, the GT with rotation angle $\xi$ is written as

$$
\begin{aligned}
G(u, v) & \triangleq G^{\xi}\{f(x, y)\}(u, v) \\
& =\iint f(x, y) \cdot K_{\xi}(x, y ; u, v) d x d y
\end{aligned}
$$

where the kernel is defined as

$$
K_{\xi}(x, y ; u, v)=\frac{1}{|\sin \xi|} \exp \left[2 i \pi \frac{(x y+u v) \cos \xi-x v-y u}{\sin \xi}\right] .
$$

Here, $(u, v)$ and $(u, v)$ are the coordinates on the input and output plane. $G(u, v)$ is the output of the GT. GT has the following special properties: The properties of the GT are summarized as follows: (1) When $\xi=0$, it corresponds to the identity transform. (2) For $\xi= \pm \pi / 2$, the GT reduces to a FT/inverse FT with a rotation of the coordinates at $\pi / 2$. (3) The inverse transform of $G^{\xi}$ is expressed as $G^{-\xi}$ or $G^{2 \pi-\xi}$. (4) The GT is periodic with $2 \pi$ and additive with respect to rotation angle, $G^{\xi_{1}} G^{\xi_{2}}=G^{\xi_{1}+\xi_{2}}$. The GT can be implemented by an optimized flexible optical system having planoconvex cylindrical lenses with a fixed distance between them. The angle $\xi$ is changed by proper rotation of two lenses. The digital GT can be implemented with two-dimensional discrete fast Fourier transform.

\section{Gamma distribution}

In probability theory and statistics, the gamma distribution is a two-parameter family of continuous probability distributions. The exponential distribution, Erlang distribution, and chi-squared distribution are special cases of the gamma distribution with different parameters [45]. A random variable $X$ that is gamma distribution with shape parameter $\alpha$ and rate parameter $\beta$ is denoted

$$
X \sim \Gamma(\alpha, \beta) \triangleq g a m m a(\alpha, \beta) .
$$

The corresponding probability density function (PDF) in the shape-rate parameterization is

$$
f(X ; \alpha, \beta)=\frac{\beta^{\alpha} x^{\alpha-1} e^{-\beta x}}{\Gamma(\alpha)},
$$

where $X>0, \alpha, \beta>0$ and $\Gamma(\alpha)$ is the upper incomplete gamma function. The upper incomplete gamma function is defined as

$$
\Gamma(s)=\int_{0}^{\infty} t^{s-1} e^{-t} \mathrm{~d} t
$$

The formula for the gamma cumulative probability function $(\mathrm{CDF})$ is

$$
F(X ; \alpha, \beta)=\frac{\gamma(\alpha, \beta X)}{\Gamma(\alpha)},
$$

where $\gamma(\alpha, \beta X)$ is the lower incomplete gamma function which is defined as

$$
\gamma(s, x)=\int_{0}^{x} t^{s-1} e^{-t} \mathrm{~d} t .
$$

If $X$ is $\Gamma(\alpha, \beta)$ random variable and the shape parameter $\alpha$ is large relative to the scale parameter $1 / \beta$, then $X$ 
approximately has a normal random variable with mean $\mu=\alpha / \beta$ and variance $\sigma^{2}=\alpha / \beta^{2}$. Figure 1 is the plot of the gamma PDF for different pairs of parameters $(\alpha, \beta)$. We also define a linear function to map the value of a random variable to the corresponding interval, as follows

$$
X^{\prime}=a X+b
$$

where $a$ is referred to as the slope of the line, and $b$ as the intercept.

\section{Encryption setup}

An optoelectronic hybrid setup of the proposed method is depicted in Fig. 2. In this scheme, the GT optical setup is constructed by using three generalized lenses with fixed distances between them. The left dotted block consists of lenses $L_{1}, L_{2}$ and $L_{1}$ denotes the first optical GT and the right one consists of lenses $L_{1}^{\prime}, L_{2}^{\prime}$ and $L_{1}^{\prime}$ represents the second optical GT. The spatial distribution of pixel value of the original image is changed digitally by the first discrete Fourier transform (DFT), attached to a gamma distribution random phase mask (RPM1), displayed on the first SLM in the input plane, and then optically transformed by the first GT. The spatial distribution of pixel value of the transformed image is changed digitally by the second DFT, attached to another gamma distribution RPM2, displayed on the second SLM in GT plane, and then optically transformed by the second GT. The encrypted complex amplitude is superimposed on the plane reference beam to produce a holographic interference fringe, which is captured and recorded as an off-axis hologram by charged couple device (CCD) camera, and digitally processed by a computer system. The encrypted complex amplitude function can be expressed as

$$
E\left(x^{\prime}, y^{\prime}\right)=G^{\xi_{2}}\left\{G^{\xi_{1}}\left\{I(x, y) \cdot \exp \left[i \phi_{1}(x, y)\right]\right\} \cdot \exp \left[i \phi_{2}(u, v)\right]\right\},
$$

where $\phi_{1}(x, y)$ and $\phi_{2}(x, y)$ are the phase functions with gamma probability distribution. $\xi_{1}$ and $\xi_{2}$ are rotation angles for the first and second GT. The decryption system has the same structure as the encryption system but uses a conjugated RPM. For real number input, the decrypted image is obtained by a reverse of the encryption process with GT at the rotation $-\xi_{1},-\xi_{2}$ and conjugation of the second RPM. In this scheme, the alignment accuracy is limited by the pixel size of $20 \mu \mathrm{m}$ and thus a large space-bandwidth product is obtained [21].

\section{Results and discussion}

Numerical simulations have been performed on a Matlab R2016a. A pepper image having $512 \times 512$ pixels and 8bits gray levels is regarded as an original image as shown in Fig. 3.

In the first case, the gamma distribution parameters are $\alpha=2$ and $\beta=1$ for RPM1 and RPM2. In order to limit most of the phase values within the required range, according to Eq. (8), the linear transformation parameters are set to $(a, b)=(1,1)$ for RPM1 and $(a, b)=(2,0)$ for RPM2. The amplitude part and phase part of the encrypted result with these parameters are displayed in Fig. 4a, b, respectively. The corresponding histograms are shown in Fig. 4c, d. The patterns of the RPMs are displayed in Fig. 4e, f. The transformation angle of the

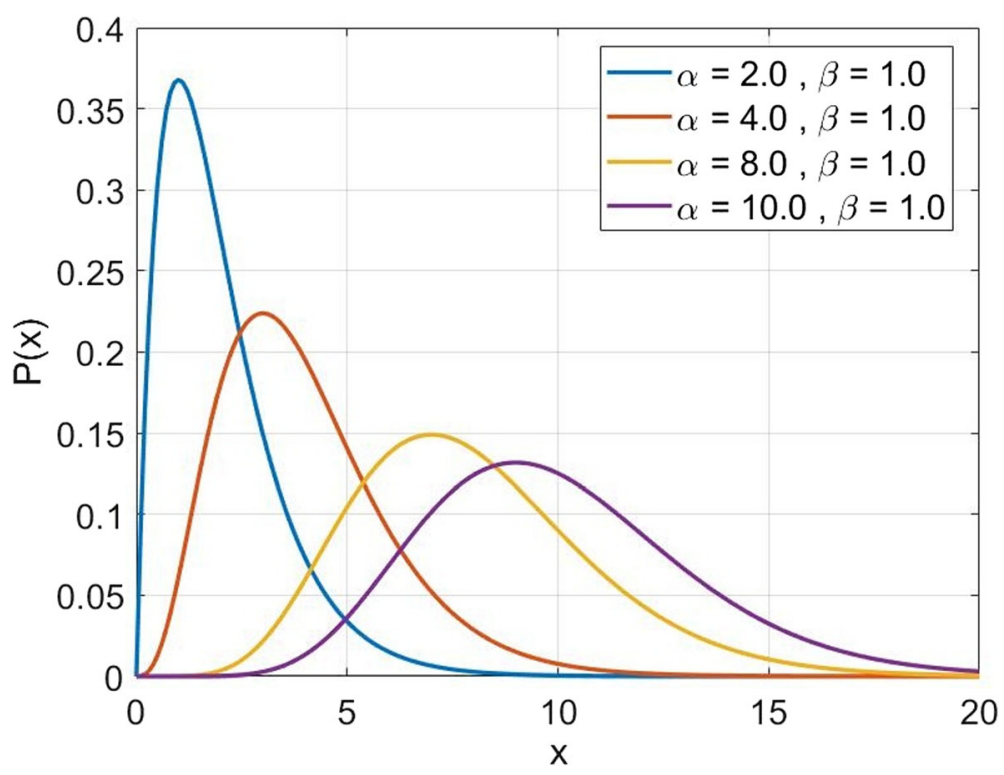

Fig. 1 Gamma PDF for different pairs of parameters $(\alpha, \beta)$ 


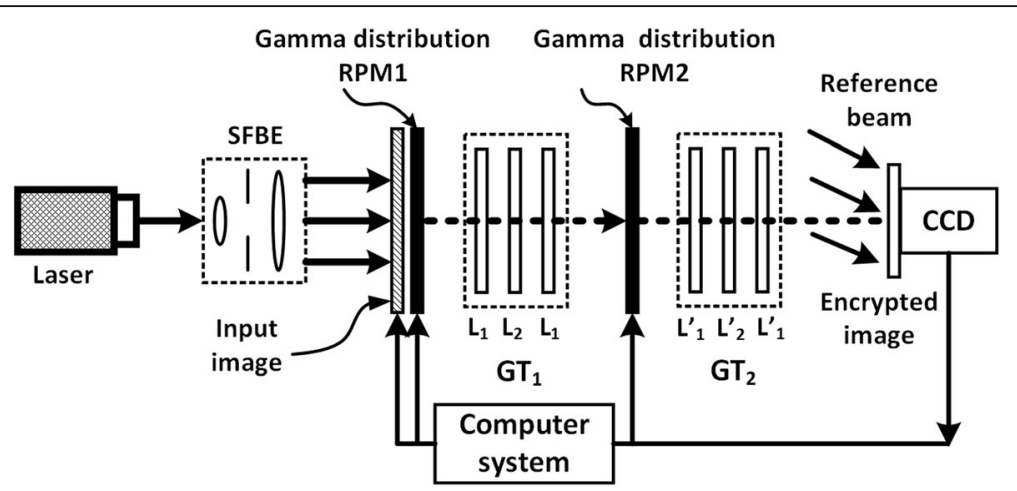

Fig. 2 Optoelectronic hybrid architecture of the proposed system

first and second GTs are both $\pi / 4$. The decrypted image with all correct keys is illustrated in Fig. 4g. For comparison, a second case is induced. A pair of uniform distribution RPMs were employed to the pepper image in GT encryption system. In this scenario, the rotation angles of GTs are $\xi_{1}=\xi_{2}=\pi / 4$. The corresponding encryption results, histograms and phase key distributions are shown in Fig. 5a-g. According to the results of hypothesis testing, under the case of a $95 \%$ confidence level, the encrypted amplitude images of both uniform and gamma distribution RPM schemes obey the Rayleigh distribution which are depicted in Figs. 4c and 5c. However, the probability distributions of encrypted phase images are significant different between two schemes. In the uniform RPM scheme, phase probability distribution obeys uniform distribution. In this case, it obeys the phase distribution model of random scattering medium.

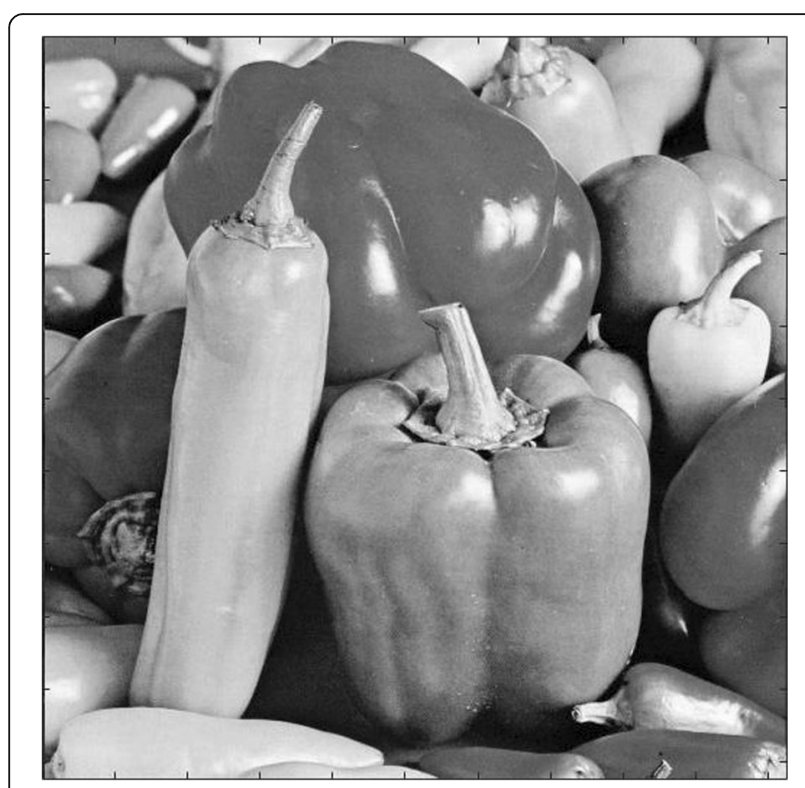

Fig. 3 Original grayscale image "pepper" with $512 \times 512$ pixels used in the numerical simulation
However, in the gamma RPM scheme, phase probability distribution does not follow uniform distribution, Gaussian distribution or Rayleigh distribution, according to the results of hypothesis testing. And output phase distribution depends on the parameter selection of gamma PDF. In fact, the proposed method is a more general way of configuring RPMs. The introduction of random distribution parameters and linear functions makes this method more flexible. On the other hand, it means that the phase distribution in the ciphertext has broken the scattering law of the random medium. It has potential to obstruct phase retrieval algorithm which is based on the hypothesis of uniform distribution of phase in the ciphertext.

To evaluate the performance of the proposed method quantitatively, there are several statistical indicators are introduced. The mean square error (MSE) and the peak signal-to-noise ratio (PSNR) are defined as

$$
\begin{aligned}
& M S E=\frac{1}{M \times N} \sum_{y=1}^{M} \sum_{x=1}^{N}\left[\left|I_{o}(x, y)-I(x, y)\right|\right] 2 \\
& P S N R=10 \log _{10}\left(\frac{R^{2}}{M S E}\right)
\end{aligned}
$$

where $I_{o}(x, y)$ and $I(x, y)$ are original and encrypted/ decrypted image respectively. $M \times N$ represents the pixels size of the image and $R=255$ is the maximum fluctuation in the input data type. The value of MSE represents the difference between input and output images at the aspect of pixel data. The quality of the decrypted result is also addressed by illustrating a retrieved image in order to assist the MSE function in vision. The decrypted result with all correct keys has the minimum of MSE which is very close to zero. The MSE values between the input image and its corresponding encrypted image is $6.1073 \times 10^{3}$ whereas the PSNR value is 10.2723. At the same time, the MSE value between the input image and its decrypted image with all correct keys is 


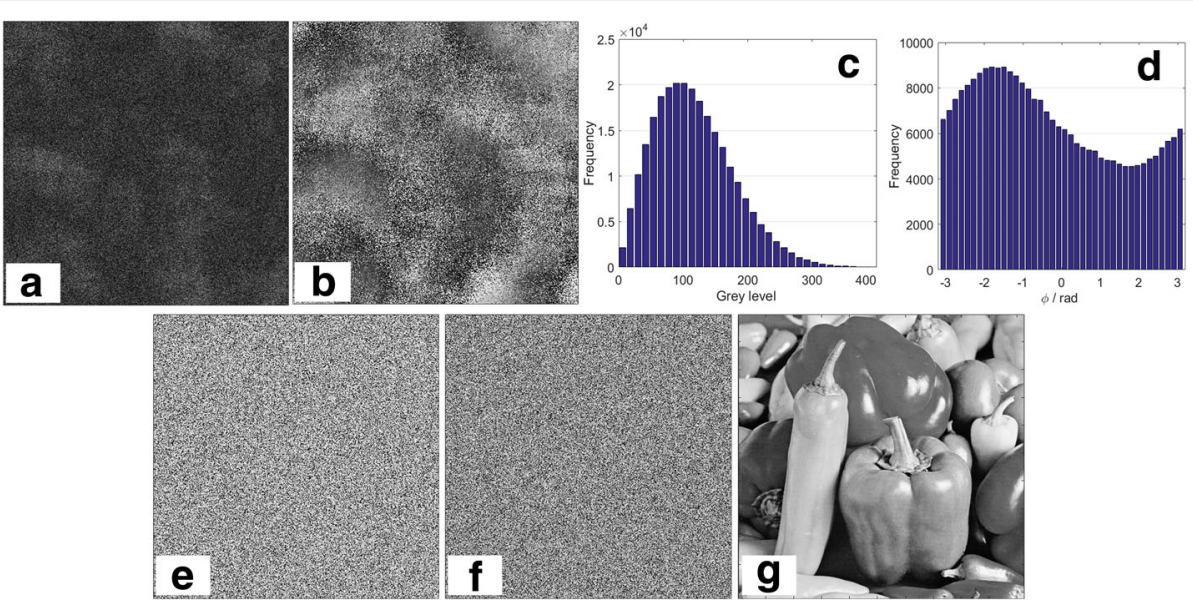

Fig. 4 Results of proposed image encryption and decryption: a Encrypted amplitude image; b Encrypted phase image; $\mathbf{c}$ histogram of encrypted amplitude; $\mathbf{d}$ histogram of encrypted phase; e first gamma distributed RPM; $\mathbf{f}$ second gamma distributed RPM; $\mathbf{g}$ decrypted image with all the correct keys

4.2097 $\times 10^{-27}$ while the PSNR value is 311.8883 . These results reveal that the original image is totally encrypted into a noise-like image and which can be reconstructed with all right key.

Further, the MSE curves between the input and their corresponding recovered images are plotted as a function of transform angles of GT1 and GT2 in gamma distribution RPM scheme and uniform RPM scheme, respectively in Figs. 6 and 7. In both the plots, MSE approaches zero when the images are decrypted with correct transform angles, whereas it increases sharply in case of a departure from the correct angles. The MSE curve of the rotation angle is not sensitive to different RPM probability models, which can be seen from the comparison results in Figs. 6 and 7. They are regarded as two sets of relatively independent encryption parameters.
The results also show that the GT scheme is very sensitive to the variations in GT angles.

Additionally, we examine the robustness of the proposed algorithm against occlusion and noise attacks. In this test, the gamma distribution parameters are $(\alpha, \beta)=(2,1)$ for both of RPMs. And the linear transformation parameters are set to $(a, b)=(1,1)$ for RPM1 and $(a, b)=(2,0)$ for RPM2. The encrypted amplitude and phase images are occluded from $20 \%$ to $80 \%$ sizes which are shown in Fig. $8 \mathrm{a}-\mathrm{h}$. The occluded pixels are replaced by zero in this test. And corresponding recovered images are displayed in Fig. 8i-l, respectively. The calculated MSE values between input and its corresponding retrieved images with all the right keys from the encrypted image with $20 \%, 40 \%$, $60 \%, 80 \%$ occlusions are $1.1310 \times 10^{3}, 3.2962 \times 10^{3}$,

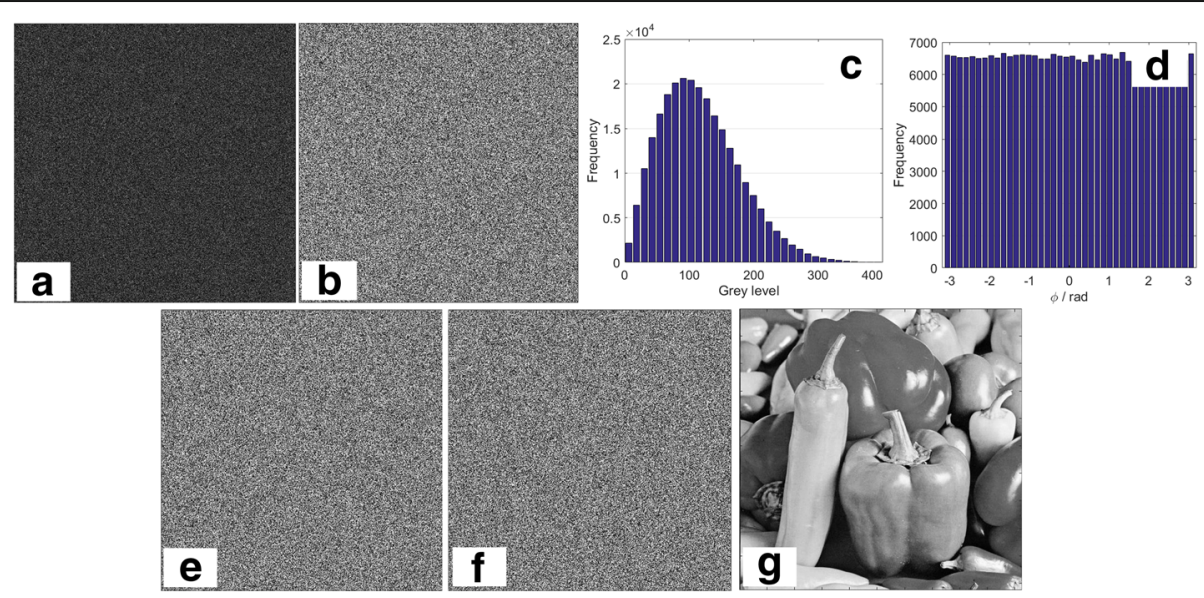

Fig. 5 Comparison results of image encryption and decryption: a Encrypted amplitude image; $\mathbf{b}$ Encrypted phase image; $\mathbf{c}$ histogram of encrypted amplitude; $\mathbf{d}$ histogram of encrypted phase; e first uniform distribution RPM; $\mathbf{f}$ second uniform distribution RPM; $\mathbf{g}$ decrypted image with all the correct keys 


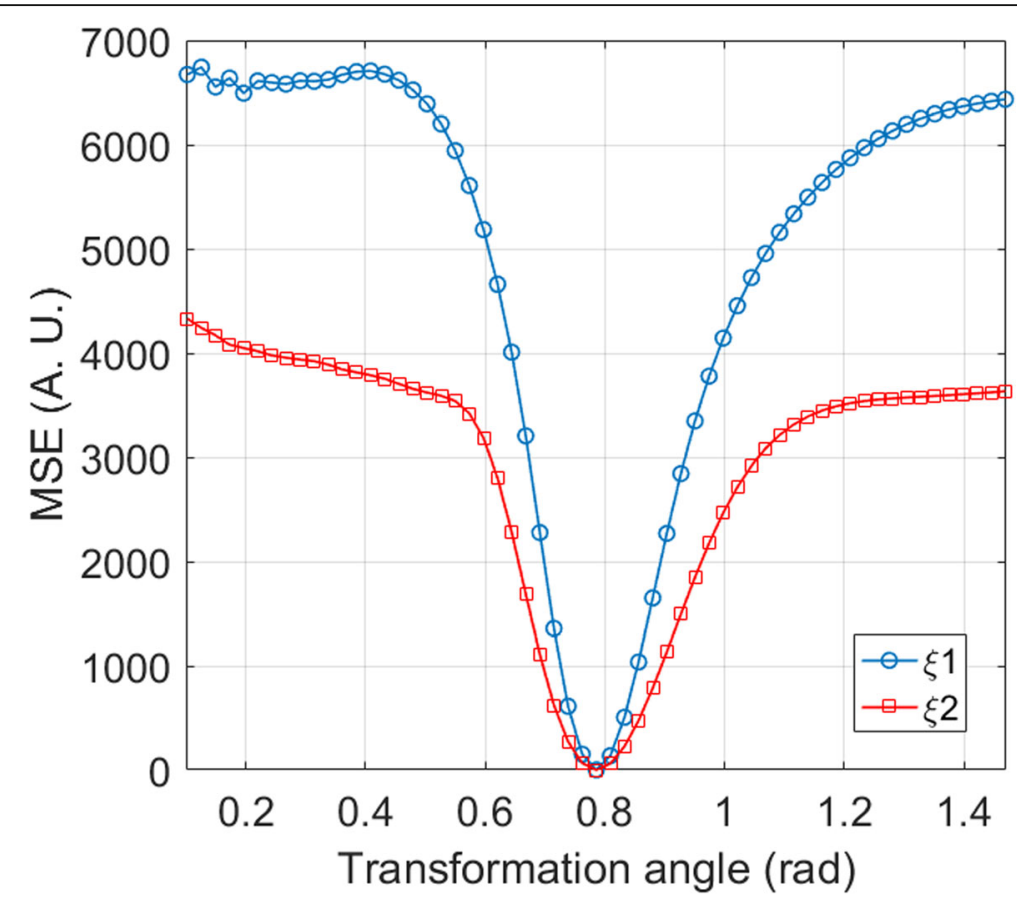

Fig. 6 MSE as a function of the transformation angle between the original image and their corresponding decrypted images in gamma distribution RPMs scheme

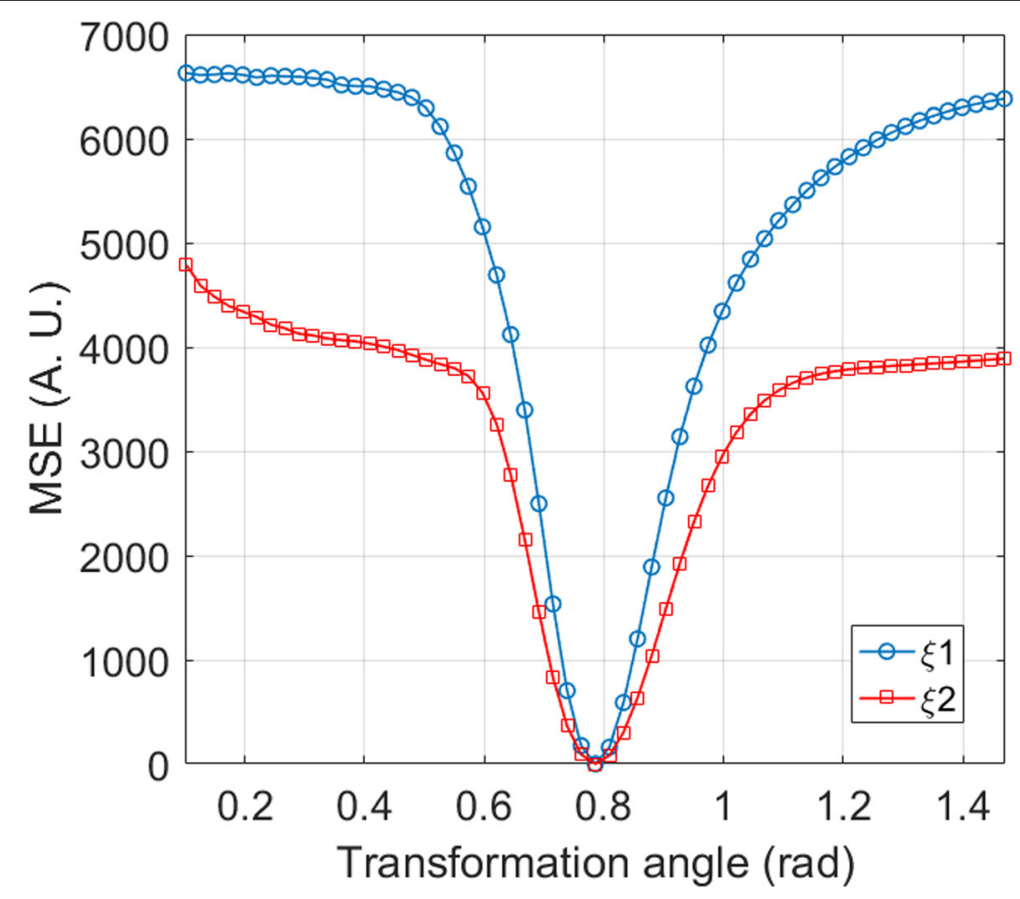

Fig. 7 MSE as a function of the transformation angle between the original image and their corresponding decrypted images in uniform distribution RPMs scheme 


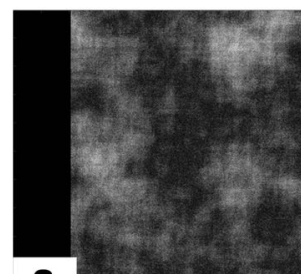

a
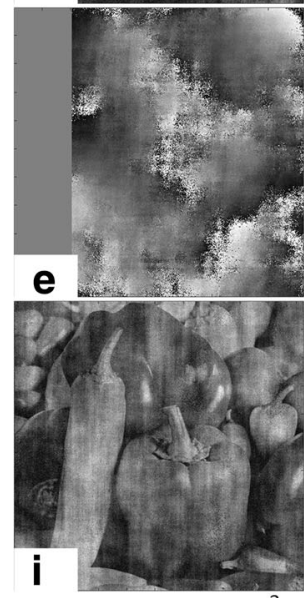

MSE: $1.1301 \times 10^{3}$

PSNR: 17.5961
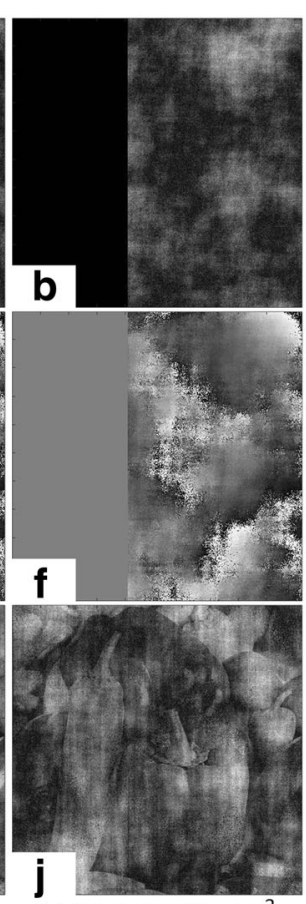

MSE: $3.2962 \times 10^{3}$

PSNR: 12.9506
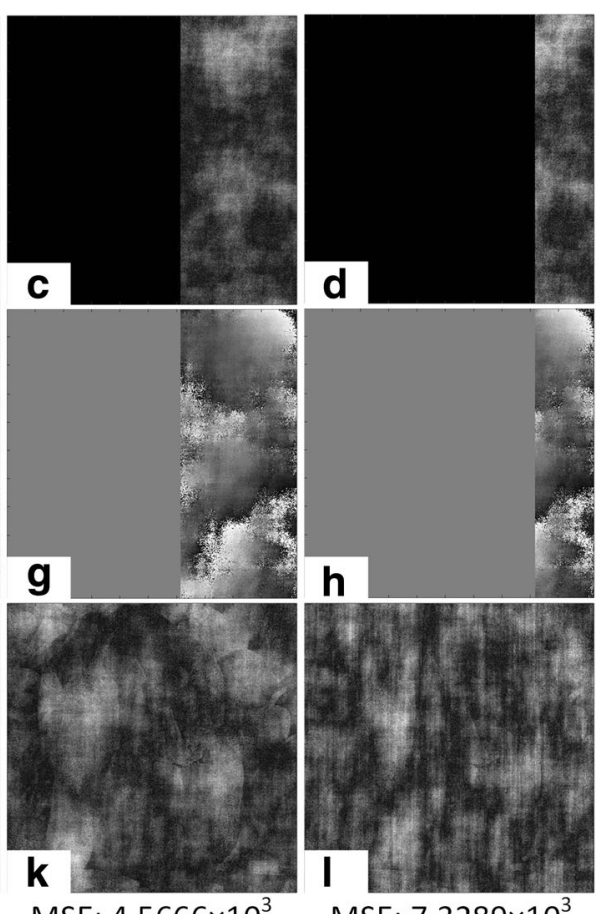

MSE: $4.5666 \times 10^{3}$

PSNR: 11.5349
MSE: $7.3289 \times 10^{3}$

PSNR: 9.4804

Fig. 8 The robustness test of the proposed method against occlusion attack on the encrypted image. $\mathbf{a}, \mathbf{b}, \mathbf{c}$, $\mathbf{d}$ encrypted amplitude images with $20 \%, 40 \%, 60 \%$ and $80 \%$ occlusion; $\mathbf{e}, \mathbf{f}, \mathbf{g}, \mathbf{h}$ encrypted phase images with $20 \%, 40 \%, 60 \%$ and $80 \%$ occlusion; $\mathbf{i}, \mathbf{j}, \mathbf{k}, \mathbf{I}$ corresponding reconstructed images from $\mathbf{a}, \mathbf{b}, \mathbf{c}, \mathbf{d}$

$4.5666 \times 10^{3}$ and $7.3289 \times 10^{3}$, respectively. The calculated PSNR values between input and its corresponding retrieved images with all the right keys from the encrypted image with $20 \%, 40 \%, 60 \%, 80 \%$ occlusions are $17.5961,12.5906,11.5349$ and 9.4804 , respectively. In the cases of $20 \%$ and $40 \%$ occluded encrypted images, their respective decrypted images with all right keys can be recognized obviously. However, the decrypted images with all correct keys from $60 \%$ and $80 \%$ occluded encrypted images, are not easily recognizable because of data loss. The proposed system shows robustness against occlusion attacks.

At the aspect of robustness analysis, the encrypted image will be checked by noise attack. The additive noise model is expressed as follow,

$$
E^{\prime}(x, y)=E(x, y)\left[1+p N_{\mu, \sigma^{2}}(x, y)\right]
$$

where $E(x, y)$ and $E^{\prime}(x, y)$ represent the ciphertexts before and after adding noise. Besides, the parameter $p$ can be regarded as a coefficient representing noise intensity. $N_{\mu, \sigma^{2}}(x, y)$ denotes Gaussian random data with the mean value $\mu$ and variance $\sigma^{2}$.

In this test, the encrypted amplitude and phase images are added Gaussian noise with different parameters. All the relevant encryption parameters are the same as in the occlusion test. The results are shown in Fig. 9a-l. From left to right columns, the noise parameters are $\left(p, \mu, \sigma^{2}\right)=(0.5,0,0.5),\left(p, \mu, \sigma^{2}\right)=(1,0,0.5),\left(p, \mu, \sigma^{2}\right)=(0.5$, $0,2)$ and $\left(p, \mu, \sigma^{2}\right)=(1,0,2)$, respectively. The MSE values from left to right each columns are $0.5236 \times 10^{3}, 1.8422 \times$ $10^{3}, 4.7273 \times 10^{3}$ and $6.5206 \times 10^{3}$, respectively. The corresponding PSNR values from left to right columns are 20.9408, 15.4775, 11.3847 and 9.9879. In the first three cases, the original image is retrieved and recognized. The quality of the retrieval image is sensitive to the encrypted phase image. The proposed system has robustness against noise attacks.

\section{Conclusions}

We have proposed an encryption scheme for grayscale images with a pair of random phase masks based on gamma distribution in the gyrator domain. The gamma distribution phase mask is preferred over the uniform distribution phase mask in order to introduce parameters that enlarge the key space. In addition to enhancing the system security, the use of gamma distribution RPM also helps in the flexibility of encryption. More importantly, the encrypted image with different parameters of gamma distribution RPMs will have different statistical 

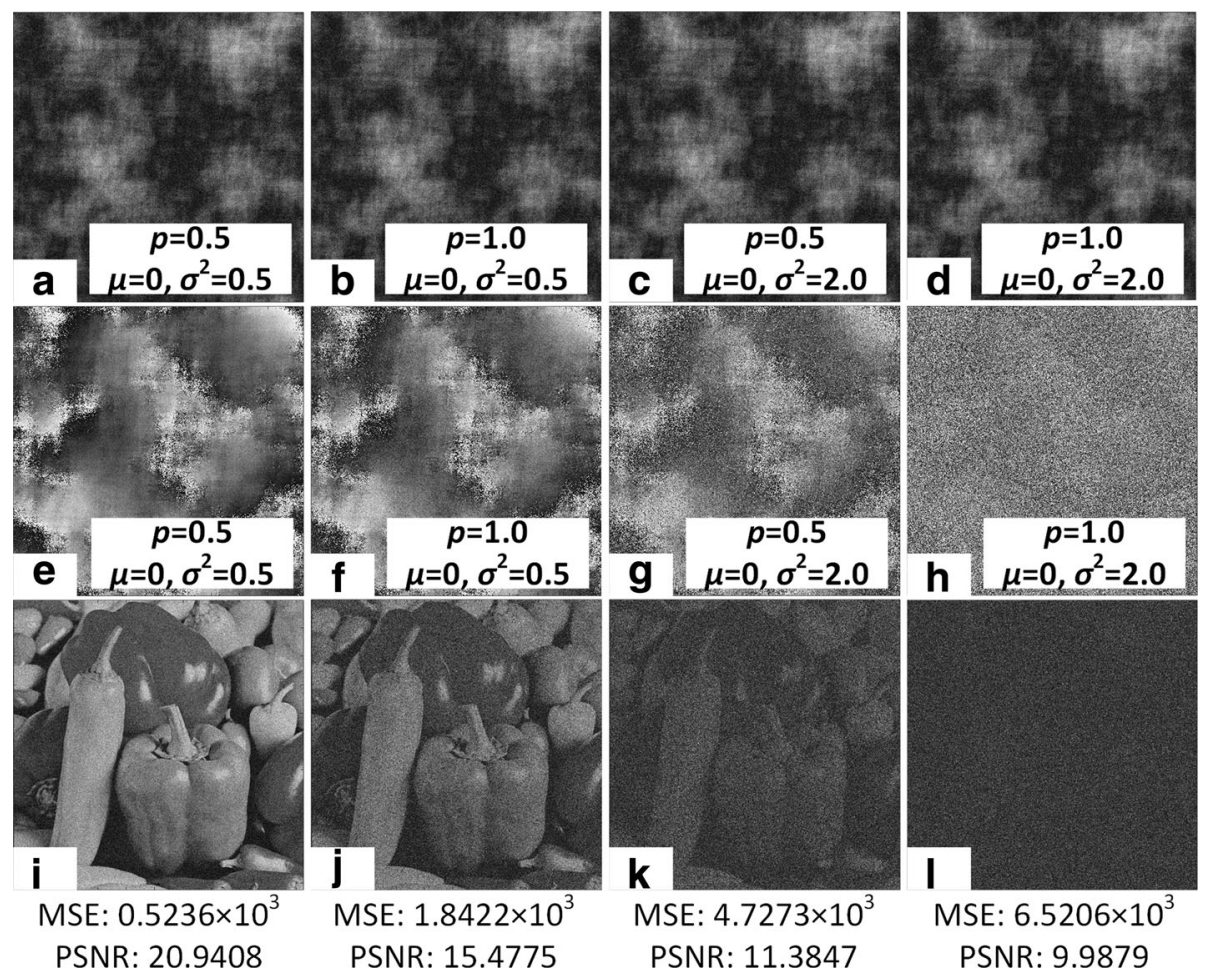

Fig. 9 Robustness test of the proposed method against Gaussian noise attack. a, b, c, d Gaussian-noised encrypted amplitude images with different parameters; $\mathbf{e}, \mathbf{f}, \mathbf{g}, \mathbf{h}$ Gaussian-noised encrypted phase images with different parameters; $\mathbf{i}, \mathbf{j}, \mathbf{k}, \mathbf{I}$ retrieved images with all the correct keys from encrypted amplitude and phase images from $(\mathbf{a}, \mathbf{e}),(\mathbf{b}, \mathbf{f}),(\mathbf{c}, \mathbf{g})$ and $(\mathbf{d}, \mathbf{h})$

distribution of phase in the ciphertext. It means that the phase distribution in the ciphertext will not obey the law of random scattering medium. Therefore, it has potential to resist the attack based on the phase retrieval algorithm. The scheme has been validated and its efficacy has been quantitatively evaluated from the computed values of MSE between input and decrypted images. The amplitude and phase pattern distribution of the encrypted image varies with the gamma distribution parameters. We have also examined the scheme's sensitivity for GT transform orders. Numerical simulations confirm that the scheme is flexible for different gamma distribution parameters. The occlusion and noise attacks analysis establish the scheme's robustness.

\section{Abbreviations \\ CCD: Charged couple device; CDF: Cumulative probability function; DFT: Discrete Fourier transform; DRPE: Double random phase encoding: FrFT: Fractional Fourier transform; FT: Fourier transform; GT: Gyrator transform; MSE: Mean square error; PDF: Probability density function; PSNR: Peak signal-to-noise ratio; RPK: Random phase key; RPM: Random phase mask; SLM: Spatial light modulator; SPK: Structured phase key}

\section{Acknowledgements}

The authors appreciate the anonymous referees for their valuable suggestions and questions.

\section{Funding}

The work reported in this article is supported by the National Natural Science Foundation of China (NSFC) (11503017); The "Summit of the Six Top Talents"
Program of Jiangsu Province (2015-DZXX-026); Jiangsu Key Disciplines of Thirteenth Five-Year Plan (20168765). The authors are grateful to Suzhou Key Laboratory for Precision and Efficient Processing Technology (SZS201712) for their support.

Availability of data and materials Not applicable.

\section{Authors' contributions}

All authors read and approved the final manuscript.

Ethics approval and consent to participate

Not applicable.

Consent for publication

Not applicable.

\section{Competing interests}

The authors declare that they have no competing interests.

\section{Publisher's Note}

Springer Nature remains neutral with regard to jurisdictional claims in published maps and institutional affiliations.

Received: 15 August 2018 Accepted: 9 December 2018 Published online: 22 December 2018

\section{References}

1. Alfalou, A., Brosseau, C.: Optical image compression and encryption methods. Adv. Opt. Photon. 1(3), 589-636 (2009). https://doi.org/10.1364/ aop.1.000589 
2. Millán, M.S.: Advanced optical correlation and digital methods for pattern matching - 50th anniversary of Vander Lugt matched filter. J. Opt. 14(10), 103001-103020 (2012). https://doi.org/10.1088/2040-8978/14/10/103001

3. Chen, W., Javidi, B., Chen, X.: Advances in optical security systems. Adv. Opt. Photon. 6(2), 120-155 (2014). https://doi.org/10.1364/aop.6.000120

4. Liu, S., Guo, C., Sheridan, J.T.: A review of optical image encryption techniques. Opt. Laser Technol. 57, 327-342 (2014). https://doi.org/10.1016/j. optlastec.2013.05.023

5. Javidi, B., Carnicer, A., Yamaguchi, M., Nomura, T., Pérez-Cabré, E., Millán, M.S., Nishchal, N.K., Torroba, R., Barrera, J.F., He, W., Peng, X., Stern, A., Rivenson, Y., Alfalou, A., Brosseau, C., Guo, C., Sheridan, J.T., Situ, G., Naruse, M., Matsumoto, T., Juvells, I., Tajahuerce, E., Lancis, J., Chen, W. Chen, X., Pinkse, P.W.H., Mosk, A.P., Markman, A.: Roadmap on optical security. J. Opt. 18(8), 083001-083039 (2016). https://doi.org/10.1088/ 2040-8978/18/8/083001

6. Vaish, A., Kumar, M.: Color image encryption using singular value decomposition in discrete cosine Stockwell transform domain. Opt. Appl. 48(1), 25-38 (2018). https://doi.org/10.5277/oa180103.

7. $\mathrm{Xu}, \mathrm{H} ., \mathrm{Xu}, \mathrm{W}$., Wang, S., Wu, S.: Phase-only asymmetric optical cryptosystem based on random modulus decomposition. J. Mod. Opt. 65(10), 1245-1252 (2018). https://doi.org/10.1080/09500340.2018.1431314

8. Ravi, K., Basanta, B., Naveen, K.N.: Nonlinear QR code based optical image encryption using spiral phase transform, equal modulus decomposition and singular value decomposition. J. Opt. 20(1), 015701-015710 (2018). https:// doi.org/10.1088/2040-8986/aa9943

9. Zhao, H., Zhong, Z., Fang, W., Xie, H., Zhang, Y., Shan, M.: Double-image encryption using chaotic maps and nonlinear non-DC joint fractional Fourier transform correlator. Opt. Eng. 55(9), 0931091-0931097 (2016). https://doi.org/10.1117/1.oe.55.9.093109.

10. Vaish, A., Kumar, M.: Color image encryption using MSVD, DWT and Arnold transform in fractional Fourier domain. Optik. 145, 273-283 (2017). https:// doi.org/10.1016/j.jileo.2017.07.041

11. Jaramillo, A., Barrera, J.F., Zea, A.V., Torroba, R.: Fractional optical cryptographic protocol for data containers in a noise-free multiuser environment. Opt. Lasers Eng. 102, 119-125 (2018). https://doi.org/10.1016/j. optlaseng.2017.10.008

12. Liu, Z., Zhang, Y., Liu, W., Meng, F., Wu, Q., Liu, S.: Optical color image hiding scheme based on chaotic mapping and Hartley transform. Opt. Lasers Eng. 51(8), 967-972 (2013). https://doi.org/10.1016/j.optlaseng.2013.02.015

13. Singh, P., Yadav, A.K., Singh, K.: Phase image encryption in the fractional Hartley domain using Arnold transform and singular value decomposition. Opt. Lasers Eng. 91, 187-195 (2017). https://doi.org/10. 1016/j.optlaseng.2016.11.022

14. Wang, J., Song, L., Liang, X., Liu, Y., Liu, P.: Secure and noise-free nonlinear optical cryptosystem based on phase-truncated Fresnel diffraction and QR code. Opt. Quant. Electron. 48(11), (2016). https://doi. org/10.1007/s11082-016-0796-3

15. Shen, X., Dou, S., Lei, M., Chen, Y.: Optical image encryption based on a joint Fresnel transform correlator with double optical wedges. Appl. Opt. 55(30) 8513-8522 (2016). https://doi.org/10.1364/AO.55.008513

16. Zhang, C., He, W., Wu, J., Peng, X.: Optical cryptosystem based on phase-truncated Fresnel diffraction and transport of intensity equation. Opt. Express. 23(7), 8845-8854 (2015). https://doi.org/10. 1364/oe.23.008845

17. Li, X., Meng, X., Yang, X., Wang, Y., Yin, Y., Peng, X., He, W., Dong, G., Chen, $H .:$ Multiple-image encryption via lifting wavelet transform and XOR operation based on compressive ghost imaging scheme. Opt. Lasers Eng. 102, 106-111 (2018). https://doi.org/10.1016/j.optlaseng. 2017.10.023

18. Liu, Q., Wang, Y., Wang, J., Wang, Q.-H.: Optical image encryption using chaos-based compressed sensing and phase-shifting interference in fractional wavelet domain. Opt. Rev. 25(1), 46-55 (2017). https://doi.org/10. 1007/s10043-017-0390-3

19. Rodrigo, J.A., Alieva, T., Calvo, M.L.: Gyrator transform: properties and applications. Opt. Express. 15(5), 2190-2203 (2007). https://doi.org/10.1364/oe.15.002190

20. Rodrigo, J.A., Alieva, T., Calvo, M.L.: Applications of gyrator transform for image processing. Opt. Commun. 278(2), 279-284 (2007). https://doi.org/10. 1016/j.optcom.2007.06.023

21. Abuturab, M.R.: Color image security system using double randomstructured phase encoding in gyrator transform domain. Appl. Opt. 51(15), 3006-3016 (2012). https://doi.org/10.1364/AO.51.003006
22. Abuturab, M.R.: Color information cryptosystem based on optical superposition principle and phase-truncated gyrator transform. Appl. Opt. 51(33), 7994-8002 (2012). https://doi.org/10.1364/A0.51.007994

23. Abuturab, M.R.: Color information security system using discrete cosine transform in gyrator transform domain radial-Hilbert phase encoding. Opt. Lasers Eng. 50(9), 1209-1216 (2012). https://doi.org/10.1016/j.optlaseng.2012.03.020

24. Abuturab, M.R.: Color information security system using Arnold transform and double structured phase encoding in gyrator transform domain. Opt. Laser Technol. 45, 525-532 (2013). https://doi.org/10.1016/ j.optlastec.2012.05.037

25. Abuturab, M.R.: Color image security system based on discrete Hartley transform in gyrator transform domain. Opt. Lasers Eng. 51(3), 317-324 (2013). https://doi.org/10.1016/j.optlaseng.2012.09.008 Abuturab, M.R.: Multiple color-image authentication system using $\mathrm{HSI}$ color space and QR decomposition in gyrator domains. J. Mod. Opt. 63(11), 1035-1050 (2015). doi:10.1080/09500340.2015.1117671

26. Liu, Z., Zhang, Y., Li, S., Liu, W., Liu, W., Wang, Y., Liu, S.: Double image encryption scheme by using random phase encoding and pixel exchanging in the gyrator transform domains. Opt. Laser Technol. 47, 152-158 (2013). https://doi.org/10.1016/j.optlastec.2012.09.007

27. Liansheng, S., Bei, Z., Xiaojuan, N., Ailing, T.: Optical multiple-image encryption based on the chaotic structured phase masks under the illumination of a vortex beam in the gyrator domain. Opt. Express. 24(1), 499-515 (2016). https://doi.org/10.1364/OE.24.000499

28. Shao, Z., Shang, Y., Fu, X., Yuan, H., Shu, H.: Double-image cryptosystem using chaotic map and mixture amplitude-phase retrieval in gyrator domain. Multimed. Tools Appl. 77(1), 1285-1298 (2017). https://doi.org/10. 1007/s11042-016-4279-0

29. Chen, H., Tanougast, C., Liu, Z., Blondel, W., Hao, B.: Optical hyperspectra image encryption based on improved Chirikov mapping and gyrator transform. Opt. Lasers Eng. 107, 62-70 (2018). https://doi.org/10.1016/j. optlaseng.2018.03.011

30. Shao, Z., Shang, Y., Tong, Q., Ding, H., Zhao, X., Fu, X.: Multiple color image encryption and authentication based on phase retrieval and partial decryption in quaternion gyrator domain. Multimed. Tools Appl. (2018). https://doi.org/10.1007/s11042-018-5818-7

31. Yadav, A.K., Vashisth, S., Singh, H., Singh, K.: A phase-image watermarking scheme in gyrator domain using devil's vortex Fresnel lens as a phase mask. Opt. Commun. 344, 172-180 (2015). https://doi. org/10.1016/j.optcom.2015.01.019

32. Tebaldi, M., Furlan, W.D., Torroba, R., Bolognini, N.: Optical-data storagereadout technique based on fractal encrypting masks. Opt. Lett. 34(3), 316318 (2009). https://doi.org/10.1364/ol.34.000316

33. Zamrani, W., Ahouzi, E., Lizana, A., Campos, J., Yzuel, M.J.: Optical image encryption technique based on deterministic phase masks. Opt. Eng. 55(10), 1031081-1031089 (2016). https://doi.org/10.1117/1.oe.55.10.103108.

34. Barrera, J.F., Henao, R., Torroba, R.: Optical encryption method using toroidal zone plates. Opt. Commun. 248(1-3), 35-40 (2005). https://doi.org/10.1016/j. optcom.2004.11.086

35. Barrera, J.F., Henao, R., Torroba, R.: Fault tolerances using toroidal zone plate encryption. Opt. Commun. 256(4-6), 489-494 (2005). https://doi.org/10. 1016/j.optcom.2005.06.077

36. Rajput, S.K., Nishchal, N.K.: Asymmetric color cryptosystem using polarization selective diffractive optical element and structured phase mask. Appl. Opt. 51(22), 5377-5386 (2012). https://doi.org/10.1364/AO.51.005377

37. Singh, H., Yadav, A.K., Vashisth, S., Singh, K.: Double phase-image encryption using gyrator transforms, and structured phase mask in the frequency plane. Opt. Lasers Eng. 67, 145-156 (2015). https://doi.org/10.1016/j. optlaseng.2014.10.011

38. Lin, C., Shen, $X$.: Design of reconfigurable and structured spiral phase mask for optical security system. Opt. Commun. 370, 127-134 (2016). https://doi. org/10.1016/j.optcom.2016.03.021

39. Lin, C., Shen, X., Lei, M.: Generation of plaintext-independent private key based on conditional decomposition strategy. Opt. Lasers Eng. 86, 303-308 (2016). https://doi.org/10.1016/j.optlaseng.2016.06.023

40. Singh, $\mathrm{H}$.: Nonlinear optical double image encryption using random-optical vortex in fractional Hartley transform domain. Opt. Appl. 47(4), 557-578 (2017). https://doi.org/10.5277/oa170406.

41. Kumar, R., Bhaduri, B.: Optical image encryption in Fresnel domain using spiral phase transform. J. Opt. 19(9), $095701-095710$ (2017). https://doi.org/ 10.1088/2040-8986/aa7cb1 
42. Abuturab, M.R.: Securing multiple information using chaotic spiral phase encoding with simultaneous interference and superposition methods. Opt. Lasers Eng. 98, 1-16 (2017). https://doi.org/10.1016/j.optlaseng.2017.05.001

43. Chen, Q., Shen, X., Dou, S., Lin, C., Wang, L.: Topological charge number multiplexing for JTC multiple-image encryption. Opt. Commun. 412, 155160 (2018). https://doi.org/10.1016/j.optcom.2017.12.015

44. Rafiq Abuturab, M.: Asymmetric multiple information cryptosystem based on chaotic spiral phase mask and random spectrum decomposition. Opt. Laser Technol. 98, 298-308 (2018). https://doi.org/ 10.1016/j.optlastec.2017.08.010

45. Papoulis, A., Pillai, S.U.: Probability, Random Variables, and Stochastic Processes, 4th edn. McGraw-Hill, Europe (2002)

\section{Submit your manuscript to a SpringerOpen ${ }^{\circ}$ journal and benefit from:}

- Convenient online submission

Rigorous peer review

- Open access: articles freely available online

High visibility within the field

- Retaining the copyright to your article

Submit your next manuscript at $\boldsymbol{\nabla}$ springeropen.com 